\title{
COLEGIO DE EDUCADORES DE PÁRVULOS DE CHILE AG
}

\section{CHILEAN PROFESSIONAL ASSOCIATION FOR EARLY CHILDHOOD EDUCATORS}

M.Soledad Rayo Quintana, Educadora de Párvulos. JUNJI. Alameda 107, piso 6 soledad.rayo@vtr.net

Mónica de Mesa Contardo, Educadora de Párvulos. CAADEM. Carlos Antúnez 1843 of. 105. contacto@caadem.cl

Gloria Laengle Scarlazetta, Educadora de Párvulos. JUNJI. Alameda 107, piso 4 glorialaengle@gmail.com

Estela Guzmán Valenzuela, Educadora de Párvulos. Colegio de Educadoras de Párvulos. Mac Iver 484 of. 38. esguval@gmail.com

Grimaldina Araya Rojas, Educadora de Párvulos. JUNJI, Dirección Nacional. Marchant Pereira 726. garaya@junji.cl

Selma Simonstein Fuentes, Educadora de Párvulos. OMEP. San Ignacio 3023. ssimonstein@gmail.com

Fernanda Salazar Acuña, Educadora de Párvulos. Universidad UCINF. Pedro de Valdivia 450. fer.salazar.1@gmail.com

Resumen: Este artículo intenta describir al Colegio de Educadores de Párvulos de Chile AG, su organización, funcionamiento y actividades realizadas en cuanto al ejercicio profesional y al desarrollo de la Educación Parvularia del país. Presenta también planteamientos de algunas colegiadas referidos al deber ser de la Reforma Educacional chilena en este nivel.

Palabras clave: reforma de educación parvularia, colegio profesional, percepciones de colegiadas. 
Abstract: This paper describes the Colegio de Educadores de Párvulos de Chile AG, its organization, operation and activities concerning professional practice and nation wide's Early Childhood Educational Development. It also presents some collegiate approaches referred to Chilean Educational Reform, at this level.

Keywords: early childhood educational reform, professional association, collegiate perceptions.

\section{IDENTIFICACIÓN}

\subsection{Misión}

Mantener una estructura y mecanismos que permitan la racionalización, protección, progreso, desarrollo, prestigio y prerrogativas de las(os) educadoras(es) de párvulos. Velar por su correcto ejercicio profesional y promover el bienestar de las(os) colegiadas(os).

\subsection{Visión}

Asegurar la interacción permanente con el medio país, especialmente con la realidad sistema educación parvularia chilena y organismos sociales, legales, económicos, jurídicos, de la nación; identificar cambios e innovaciones que pudieran afectar las tareas programadas y visualizar, proponer y desarrollar alternativas de apoyo y promoción de los propósitos de la organización, en el contexto nacional.

\subsection{Propósito}

Está centrado en el colegiado y las relaciones a mantener con las instituciones universitarias formadoras de educadores, el ambiente profesional, cultural y la sociedad en general: 
a) En cuanto al colegiado, se pretende cautelar su desempeño profesional, entregarle apoyo en su profesión, protección legal y la asistencia laboral que necesite.

b) Mantener el contacto permanente con instituciones universitarias formadoras de educadoras(es) y el medio cultural en general para retroalimentar el quehacer gremial, y estimular su dinamismo, pertinencia y vigencia.

c) Colaborar con el contexto educacional nacional a través de asesorías y orientaciones a las diversas entidades que lo soliciten.

d) Ofrecer y desarrollar actividades de difusión y estudio de la Educación Parvularia y su compleja problemática, tanto en períodos de cambios e innovaciones como en tiempos de actividad regular de la vida profesional.

e) Proponer, fundamentadamente, de acuerdo a los Estatutos (1977) las modificaciones legales a proyectos de Educación Parvularia (que fueren necesarias) en cuanto a la atención optimizada de las niñas(os) menores de seis años, al personal profesional y técnico que les atiende y a la participación de padres y comunidad en el proceso educativo sistemático que se desarrolla en los respectivos jardines infantiles y salas cuna (Estatutos del colegio de Educadoras, 1977).

\subsection{Estructura organizacional del Colegio}

El actual directorio nacional asumió en junio de 2013. Este grupo de profesionales trabajan en distintos ámbitos de la Educación Parvularia, lo que lo hace representativo del colectivo que lo constituye.

\section{Directorio Nacional Período 2013-2016:}

- Presidenta: María Soledad Rayo Quintana

- Vice Presidenta: Verónica Guzmán Karrer 
- Secretaria: Gloria Laengle Scarlazetta

- Tesorera: Estela Guzmán Valenzuela

- Directoras: Erna Castro Pino

Mirta Muñoz Manríquez

Mónica de Mesa Contardo

Los objetivos de la gestión están explícitos en los estatutos que fueron elaborados en 1983, tiempos nada fáciles para los gremios. Posteriormente, fueron modificados en el año 1997. Reconocen, dada las circunstancias políticas nacionales, la importancia de las profesionales que hubieren estudiado en el extranjero o retornado al país, como también al profesor(a) normalista con mención en Educación Parvularia, puesto que en toda esta gama de formación se produce una invaluable transferencia de experiencias, conocimientos y aportes a la profesión.

En estos estatutos se establece, además, que podrán colegiarse alumnas(os) regulares que se encuentren estudiando el último año de la carrera y aquellas(os) en vías de titulación. Lo anterior con membrecía transitoria. Para resguardar el ejercicio legal de la profesión se detalla que la transitoriedad de la membrecía no acredita ni habilita el ejercicio profesional. De no cumplirse con los requisitos en el período establecido para la titulación, ésta se pierde.

Convocar a la reflexión, recoger inquietudes y propuestas, dar respuesta a las necesidades de socias y socios, participar en las diferentes actividades de la sociedad civil y, además, vincularse con los poderes del estado, ejecutivo y legislativo, implican una permanente disposición y energía para ponerla al servicio del gremio.

Este Colegio AG ha participado en todas las instancias a las que ha sido convocado, mostrando interés y compromiso en las políticas públicas vinculadas a 
la Educación Parvularia, sus representados, la carrera docente, la formación de sus profesionales y las condiciones laborales de estos.

En forma permanente se organizan seminarios y otras actividades en articulación con otras instituciones y organismos. Todos ellos de acuerdo a los intereses de nuestras socias y socios: el sistema, la sociedad y la Educación Parvularia, alcanzando niveles de calidad significativos y con una alta convocatoria.

Para apoyar los propósitos de esta organización se cuenta con el siguiente equipo complementario de trabajo:

- Secretaria (permanente)

- Periodista (por periodo)

- Abogado (permanente)

- Contador (permanente)

- Encargado de pagina web (permanente)

- Asistente de aseo (permanente)

\section{ACTIVIDADES REALIZADAS Y LOGROS.}

Entre las numerosas y variadas actividades realizadas por este Colegio A.G., figuran las siguientes:

- Entrega de premios a aquellas educadoras(es) de párvulos que se hayan destacado en su ejercicio profesional, las cuales son postuladas por sus respectivas comunidades educativas. Existen premios que se otorgan regularmente y otros que son motivados por situaciones especiales.

- Organización de seminarios nacionales y regionales sobre temas de actualidad en Educación Parvularia. 
- Desarrollo de tertulias y conversatorios fundamentados sobre problemas vigentes del niño menor de 8 años, abiertas a la opinión y participación directa de educadoras(es).

- Consultas legales y académicas de colegiadas(os) sobre su situación laboral o problemas de organización y administración curricular.

- Apoyo técnico a instituciones vinculadas a la Educación Parvularia.

- Reuniones y entrevistas con autoridades y representantes del sistema educativo; participación en comisiones y mesas de estudio y discusión.

- Renovar colegios regionales, comunales y capítulos profesionales.

- Gestionar nuevos proyectos ya aprobados, como: sede propia, casa de acogida a colegiadas(os).

- Editar boletín y revista de la organización.

- Mantener convenios con instituciones diversas relacionadas con la Educación Parvularia y con el bienestar de las colegiadas(os).

- Participación en consejos oficiales.

- Otros.

\subsection{Distinciones anuales a las Educadoras de Párvulos a nivel nacional}

En el marco de la celebración del "Día de la Educación Parvularia y de la Educadora de Párvulos", este Colegio Profesional premia cada año a las(os) educadoras(es) que, a juicio de sus pares o comunidad educativa, sean merecedoras de un reconocimiento por su destacada labor y aporte a la Educación Parvularia a nivel nacional, regional 0 local. 
Dentro de esta distinción existen cuatro categorías, las cuales detallamos a continuación:

Premio Carmen Fischer Ramírez: Se otorga al profesional de Educación Parvularia que se haya destacado por sus prácticas pedagógicas y ese trabajo de excelencia se vea reflejado en los logros de sus niños y niñas. Este profesional ha sido un motor permanente de estimulación de nuevos y mejores aprendizajes, formador de valores y principios éticos en los párvulos a su cargo, colaborador con la gestión integral del jardín infantil o colegio, demostrando un liderazgo eficiente y eficaz con su equipo de trabajo y con los padres y apoderados.

Este premio fue creado en 1995 y, a la fecha, se ha otorgado a 20 educadoras de párvulos.

Premio Linda Volosky de Cabello: Se otorga al profesional de Educación Parvularia que se haya destacado por realizar un aporte significativo a la investigación educativa, tanto en la teoría como en la práctica de la educación inicial a nivel nacional.

Dichas investigaciones deben haberse centrado en los párvulos como sujeto principal de ellas y su contexto educativo: características de los niños chilenos en sus diferentes etapas de desarrollo, infraestructura, organización y administración de jardines infantiles y salas cuna, programas educativos diversos, formación y desempeño de educadores(as) de párvulos en el aula, diferentes enfoques curriculares, entre otros.

Este premio fue creado en 1995 y, a la fecha, ha sido otorgado a 20 educadoras de párvulos.

Premio Rebeca Soltanovic de Stein: Se otorga al profesional de la Educación Parvularia que se haya distinguido por su calidad docente en la formación de los futuros educadores y por la actualización e innovación de su ejercicio profesional, constituyendo un modelo a seguir por parte de sus discípulos. Lo anterior, le ha permitido generar profesionales conscientes de la importancia de adquirir una 
excelente formación académica, en un marco ético, moral, social y motivacional para el mejoramiento de la Educación Parvularia o inicial en nuestro país.

Este premio fue creado el año 2001 y, a la fecha, lo han recibido 14 educadoras de párvulos.

Premio Preservación de la Cultura de los Puebles Originarios: El Colegio de Educadores de Párvulos de Chile $A G$, ha querido distinguir a aquellas profesionales que se destaquen por su contribución a la difusión, desarrollo y valoración de la cultura, identidad y aportes de los pueblos originarios.

Este premio fue creado el año 2010. Hasta el momento se ha otorgado a seis profesionales

Distinciones especiales otorgadas por situaciones específicas, a profesionales de la educación

Entre estos, se ubica el premio Bicentenario de la Nación, el cual fue entregado el año 2010 a diez profesionales.

El año 2011 se reconoce el aporte a la Educación Parvularia de la psicóloga educacional Neva Milicic Müller.

\subsection{Organización de seminarios nacionales y regionales}

Dentro de las actividades que este Colegio Profesional realiza durante el año, destaca la organización de seminarios donde se tratan diversas temáticas vinculadas a la Educación Parvularia.

En estas instancias, que tienen como objetivo la participación y el intercambio de ideas, figuran distintos actores de la educación inicial, los cuales se reúnen en un contexto de retroalimentación entre el público y los expositores.

Entre estos seminarios, se puede mencionar: 
- Los realizados en colaboración con la U Mayor: "Apoyo a la Gestión de Calidad en los Jardines Infantiles y Salas Cuna" y "Evaluación Auténtica en Educación Inicial" (agosto, ambos en el año 2009).

- "Innovación Pedagógica Reggio Emilia", en colaboración con JUNJI y Universidad Finis Terrae (2009).

- "Implicancias del Primer Kindergarten Fiscal en el Desarrollo de la Educación Parvularia en Chile", lo que requirió una verdadera investigación histórica realizada por el Colegio de Educadores de Párvulos de Chile AG (2006).

- "Educación Parvularia en el siglo XXI", con la colaboración de destacados profesionales que culminó con una publicación, la que incorporó las presentaciones de los especialistas participantes (marzo, 2008).

- También se puede mencionar el "Seminario de Políticas Públicas para el Bicentenario" en el que participaron organismos tales como Mineduc, Mideplan, Sernam, Minsal, JUNJI, Dirección del Trabajo y destacados parlamentarios (2009).

- En el año 2014, en el marco de la promoción de la capacitación a nivel regional, se ejecutaron dos seminarios, tanto en Coyhaique como en Punta Arenas, con el patrocinio de instituciones públicas y la organización gremial Aprojunji.

- Otro de estos eventos se llevó a cabo el miércoles 12 de noviembre del año 2014 en Santiago y tuvo por título "Jornada de Trabajo por la Educación Parvularia: Desde la Propuesta de Reforma Educacional a las Prácticas de Aula", actividad realizada en conjunto con el capítulo de docentes, universitarias y otros organismos. 
Durante aproximadamente nueve horas, en el Auditorio de la Universidad de las Américas, se formaron siete mesas de discusión integradas por educadores, técnicos, directores, sostenedores, estudiantes, académicos y otros profesionales ligados la educación infantil.

En cada una de estas mesas de discusión se trató un tema en específico, contando con un moderador y un secretario que finalmente entregó las conclusiones de cada grupo de debate. Se abordaron tópicos como:

- La nueva institucionalidad en Educación Parvularia.

- Profesión docente y proyecciones laborales de educadores de párvulos.

- Rol de las universidades en la Educación Parvularia.

- Situación actual de la formación de técnicos en Educación Parvularia.

- Niño sujeto de derechos.

- Prácticas docentes activas en la Educación Parvularia.

- Sistema de protección de la infancia hoy.

Esta jornada, que contó con un marco de público cercano a las 300 personas, sirvió para generar múltiples propuestas en base a un trabajo conjunto y articulado entre los participantes.

\subsection{Tertulias}

Reuniones realizadas con educadores de párvulos para analizar problemas específicos que preocupan a la profesión, unificar criterios y compartir opiniones sobre temas candentes y actuales. Importa, además, el debate y el contraste de ideas y opiniones. 
El actual directorio ha organizado dos tertulias, a las que asistieron profesionales de diferentes universidades.

La Primera Tertulia se efectuó el 11 de Junio de 2014, en el Salón Auditorio de la Universidad SEK de Santiago con un nutrido grupo de especialistas en la materia.

Presidida por María Soledad Rayo y moderada por la Directora Mónica de Mesa en conjunto con la socia Ximena Arriagada, se dio curso a esta Primera Tertulia con el objetivo de provocar un intercambio de opiniones a fin de poder concluir respecto a lo que, como especialistas, se piensa acerca de la nueva legislación sobre Educación Parvularia.

Participaron aportando visiones al respecto profesionales de las Universidades SEK, UCINF, JUNJI, Fundación Elige Educar, Academia Montessori, Red Solare y Chile Crece Contigo.

Se planteó, entre otros temas, la necesidad de valorar y hacer valorar la profesión, lograr consensos respecto a calidad y fiscalización, así como tener una firme postura frente a la no escolarización de la Educación Parvularia.

Para lograr lo señalado precedentemente, se concluye que los especialistas del área deben pasar de la invisibilidad a la visibilidad de sus planteamientos y, por ello, será menester seguir perfeccionando este tipo de iniciativas del Colegio e impulsar este Ciclo de Tertulias.

La Segunda Tertulia, que se realizó el 12 de agosto del 2014 en las dependencias de la Universidad SEK, estuvo referida a generar participación y diálogo relacionado con la importancia de ser parte, como profesionales, del proceso que conlleva la propuesta de Reforma de la Educación Parvularia que está en marcha respecto a este nivel educativo en nuestro país.

Participaron representantes de las universidades: SEK, Ucinf, Santo Tomás, Andrés Bello, Universidad de las Américas, Academia Montessori y profesionales en general. 
Los temas analizados fueron los contenidos trabajados en las mesas de discusión del Mineduc: calidad de la educación, evaluación de la Educación Parvularia, nueva institucionalidad y carrera docente.

\subsection{Consultas de Educadoras.}

Durante el año 2014, se realizaron diversas labores relacionadas con esta área: El Colegio de Educadores de Párvulos de Chile AG se presenta como una opción para recibir consultas y realizar asesoría jurídica a aquellas educadoras(es) de párvulos colegiadas a lo largo de todo el territorio nacional. Se realizaron, específicamente, asesorías concernientes a sumarios administrativos, consultas acerca de condiciones laborales, sistemas de pensiones, contratos de trabajo e irregularidades en seguros sociales de educadoras(es) que, a pesar de tenerlos, no podían hacerlos valer efectivamente.

También se llevan a efecto estudios técnicos-legales, respeto de los proyectos de ley emanados del Congreso Nacional o del poder Ejecutivo. Se realizaron, además, tres presentaciones en total en las Comisiones de Educación y Hacienda, tanto de la Cámara de Diputados como del Senado. Por otra parte, se realizaron presentaciones respecto a temáticas jurídicas relevantes relacionadas a la educación de párvulos y al derecho a la educación. Dentro de estas presentaciones se encuentran, entre otras:

I. Exposición del Colegio de Educadores de Párvulos para el seminario "Reforma y educación inicial", en donde se ponía énfasis principalmente en la necesidad de crear proyectos que respondieran al propósito de la Educación Parvularia, es decir, orientados a conseguir sus fines y a la falta de democracia que hay actualmente en la generación de dichos proyectos, proponiendo el Colegio aportes para la articulación y definición de la propuesta. 
II. Minuta con análisis crítico de la Reforma de Educación Parvularia.

III. Publicación en la revista "Docencia" de dos documentos sobre las condiciones laborales de los educadores de párvulos.

\subsection{Apoyo técnico a instituciones vinculadas a la educación.}

El Colegio de Educadores de Párvulos de Chile, revisa, orienta, apoya o rechaza los contenidos pedagógicos en libros, cuadernos para el nivel transición, materiales didácticos y juguetes para los niveles educativos como niveles medio y salas cuna en empresas que solicitan su patrocinio.

Se otorgó patrocinio al libro "NICOLÁS TIENE 2 PAPÁS", publicación que provocó reacciones negativas dada la realidad sociocultural y valórica existente en nuestro país, tema que no se ha abordado en profundidad, el cual implica la aceptación de la diversidad de familias presentes en la sociedad actual.

\subsection{Otras actividades}

- Participación en reuniones a las Comisiones de Educación y Hacienda del Senado y Cámara de Diputados.

- Participación en la mesa de discusión convocada por el Mineduc para la Reforma de la Educación Parvularia.

- Participación en la discusión del Plan Maestro referente a la Carrera Docente desde la visión de la Educación Parvularia.

- Reuniones con diversas autoridades y organizaciones del Estado para clarificar temáticas sobre la Reforma de la Educación Parvularia.

- Gestionar la renovación de los Colegios Regionales articulando la formación de directorios comunales, regionales, capítulos institucionales, etc. 
- Gestionar, ante bienes nacionales, un comodato de bien raíz para sede y Casa de Acogida de Educadores(as) de Párvulos.

- Potenciar la incorporación y participación de educadoras(es) de párvulos, especialmente las(os) recién tituladas(os) o egresadas(os).

- Editar la publicación de la revista del gremio.

- Mantención de convenios con universidades abocadas a la formación, capacitación y perfeccionamiento de profesionales de la Educación Parvularia.

- Edición del boletín informativo del colegio para este periodo de gestión.

- Participación de integrantes del Directorio Nacional en diversos eventos organizados por JUNJI, universidades, Junaeb, Federación de Colegios Profesionales e instituciones públicas, entre otros.

- Participación en el Consejo de Sociedad Civil del Ministerio del Trabajo y Seguridad Social (Ley 20.500)

\section{PERCEPCIONES DE ALGUNAS EDUCADORAS DE PÁRVULOS COLEGIADAS}

Los temas que destacan en sus reflexiones son:

- Reforma Educativa.

- Formación Profesional.

- Principios de la Educación Parvularia.

- Identidad y profesionalismo.

Los principios que sustenta el enfoque de los Derechos de la Infancia son los que se deben tener en cuenta en la planificación de la educación en la infancia temprana. Estos principios son: 
La calidad, la cual se refiere, en un sentido importante, al aumento de cobertura en las poblaciones de menores recursos económicos, orientada a entregar una educación adecuada de niñas y niños. Brindarles oportunidades a los más pequeños, donde el desarrollo temprano de sus capacidades, habilidades y destrezas, deben ser potenciadas con mayor precisión debido a los procesos evolutivos que van viviendo en tan cortos períodos de tiempo.

La equidad que alude al acceso de oportunidades: la educación debe partir desde las edades más tempranas para superar la falta de oportunidades socioeconómicas y culturales, donde el desarrollo integral, como inicio al despertar de la inteligencia, es fundamental. Es necesario favorecer este desarrollo desde sus comienzos y en forma profesional.

La flexibilidad y diversidad referidas a la variedad cultural, étnica, de religión, de situación física y/o mental, entre otros. Se trata que la educación los aborde con apertura a todos y todas sin importar las diferencias, con un sentido de enriquecimiento educativo.

La participación de las familias y los padres en el proceso educativo sistemático es esencial: el sistema debe promover su integración desde el inicio del proceso formativo y favorecer el desarrollo de competencias parentales.

La pregunta es ¿cómo articular las transformaciones que se requieren para que la Educación Parvularia cobre sentido en relación a los principios que sustentan, fundamentalmente, el enfoque de los Derechos de la Infancia? Su respuesta positiva la designaría como una Educación Parvularia de calidad.

Algunas de las condiciones serían las siguientes: 


\section{I.- Fortalecimiento de la Educación Parvularia.}

1.-Equidad, una oportunidad de atención integral para todos y todas.

a) Responsabilidad del estado de proporcionar las oportunidades de financiamiento para cumplir con la normativa vigente estipulada por la Intendencia de la Educación Parvularia en cuanto a infraestructura, recursos didácticos, coeficiente de personal, ratio, entre muchas otras características.

b) Una Educación Parvularia integral, considerando al niño y niña desde su diversidad, respetando su origen étnico, extranjero, religioso, social, cultural y necesidades educativas especiales.

2.- Educación Parvularia Universal.

Responsabilidad del Estado de proporcionar gratuidad y libre acceso a los niños y niñas menores de seis años que así lo requieran para los niveles de atención que les correspondan, según su edad, sin selección alguna de los educandos, sin obligatoriedad.

\section{II.-Formación Profesional.}

Selección de las o los postulantes a estudiar la carrera de Educación Parvularia, considerando aspectos tales como: salud física y psíquica, lenguaje oral y escrito, psicomotricidad gruesa y fina, características de creatividad, valores socioculturales y otros que la acreditarán para un desempeño profesional adecuado.

Los estudiantes de la carrera de Educación Parvularia deben demostrar estándares profesionales, mediante prácticas en aula con acompañamiento tutorial, con el fin de asegurar un desempeño eficiente.

Además, deben conocer las materias necesarias y actualizar los conocimientos que les permitan desempeñarse adecuadamente.

Instituciones educacionales y centros de capacitación deben facilitar el acceso al perfeccionamiento, asegurando una educación continua. Tanto estudiantes como 
profesionales de la Educación Parvularia deben cautelar el ejercicio ético de su profesión, tomando en cuenta que el niño es un sujeto de derechos y que el profesional es responsable frente a la sociedad civil en cuanto a su tarea.

\section{Condiciones laborales del Educador de Párvulos.}

Se requiere:

- Una remuneración de acuerdo a la labor educativa que realiza y a la especialización lograda, además de considerar los cargos que se desempeñan, de acuerdo a las normativas de una carrera docente actualmente en análisis.

- Jornadas laborales de no más de 8 horas pedagógicas diarias, considerando horas semanales para evaluar y planificar. GLS

En el contexto de la actual reforma que contempla la creación de una Subsecretaría de Educación Parvularia encargada del diseño de las políticas públicas en la materia y una Intendencia de Educación Parvularia, no solo se está relevando el nivel en el sistema educativo, sino también la función de la profesional Educadora de Párvulos.

Según Alvaro Marchesi( ${ }^{2}$, una de las dimensiones identificadas para una educación de calidad es la valoración social de la infancia, aspecto que queda de manifiesto en las políticas que concretan un cambio estructural para la Educación Parvularia, lo que permitirá focalizar y observar en su real dimensión lo específico, propio y distintivo del nivel.

Los discursos políticos aluden a rasgos positivos y potencialidades que tiene la infancia. Los niños y niñas son considerados sujetos de derecho y observados como el futuro de la sociedad o país. Se habla del retorno de la inversión en 
cuanto al presupuesto invertido en la infancia, el cual tiene un gran impacto a largo plazo, alcanzando mejores niveles de desarrollo humano y social en el país.

La tan ansiada calidad está, sin duda, determinada de manera importante por el liderazgo de la educadora de párvulos en la gestión pedagógica, los niveles de apropiación de su rol, su capacidad argumentativa, crítica y reflexiva en la toma de decisiones. El cambio estructural esperado en el marco de la reforma en curso puede ser una ventana de oportunidades para el nivel y la educadora de párvulos que, claramente, necesita fortalecer su valoración ética y social, y también transitar hacia el empoderamiento profesional.

Ya no existe discusión respecto a la importancia de la edad temprana ni del retorno de la inversión en la primera infancia. Sin embargo, la valoración de la profesional especialista en niños y niñas menores de seis años aún no alcanza el reconocimiento, estatus social y económico coherente con la relevancia del nivel. El discurso político no se condice con la valoración del ejercicio de la función y la complejidad de ésta.

Existe en el ser humano una edad óptima o de mayor sensibilidad a los distintos tipos de estímulos, pasado el cual, las condiciones no vuelven a ser iguales. Es así como la cantidad, calidad, intensidad y diversidad de las experiencias ( ${ }^{3}$ ) deben ser cuidadosamente seleccionadas y mediadas por un profesional en el área. Esta afirmación es la clave de la especialización, valoración y profesionalización de la educadora de párvulos.

La academia aumenta su preocupación por la formación de educadoras de párvulos en referencia a los estándares establecidos para su egreso, el marco para la buena enseñanza y los sistemas de acreditación de la carrera, respectivamente. Mientras que en el contexto laboral se enfrenta a sistemas de acreditación de la calidad de la educación, perfiles de competencias y sistemas 
de calificación de desempeño. Cada día son más las expectativas y rigurosas las exigencias.

Finalmente, es importante señalar que tal como lo define un estándar para la formación de la educadora de párvulos, se espera que la profesional aborde la dimensión ética y moral de su profesión, a través del compromiso con su propio aprendizaje y con el aprendizaje y formación de sus párvulos. De este modo, se aspira a una profesional capaz de aprender en forma continua, conocer cómo se genera y transforma la cultura escolar, y estar preparada para promover el desarrollo personal y social de las niñas y niños a su cargo". GAR

Se requiere que todos quienes están en la Educación Parvularia se revaloricen. Las neurociencias dan un fuerte sustento para mostrar la relevancia de este trabajo. Se preguntan ¿por qué la Educación Parvularia es percibida como el nivel más bajo del sistema educacional? Puede haber muchas respuestas: no es un nivel obligatorio, participan mayoritariamente mujeres. Sin embargo, la Educación Parvularia tiene algo que decir. No es un problema que se va a resolver mañana, pero es necesario proponerse combatir los prejuicios y la ignorancia.

Se debe romper los modelos tradicionales de la Educación Parvularia que se confunden con un rol maternal. El lenguaje de los y las educadoras de párvulos debe reflejar el conocimiento que les es propio. Es necesario validar el rol profesional del educador de párvulos tal como se hace con el de otros profesionales.

Sin duda, construir una imagen positiva, diferente a la actual es algo que requiere del apoyo del gobierno. Y ello no es tarea fácil. Lo económico y lo ideológico no es lo único que redefine el campo profesional. Se debe poseer firmes principios éticos que conduzcan hacia lo que es mejor para los niños y niñas. Saber teoría de la ética no es ser ético. Ingrid Pramling, ex presidenta mundial de la OMEP, plantea 
que la enseñanza de problemas que se están investigando podría ser una estrategia apropiada en la formación de educadores. Es una manera de aproximarse a la teoría desde la práctica porque el conocimiento no garantiza el actuar correcto. Se requiere reflexionar, aprender a problematizar, a dejar atrás los prejuicios. Dejar de pensar en el niño solo de 0 a 6 años; sino considerarlo desde antes del nacimiento y hasta después de su entrada a la escuela. Examinar el concepto de infancia del siglo XXI, como el punto de partida de análisis y reflexión crítica.

Se requiere re-conceptualizar el campo de la Educación Parvularia y los procesos de globalización que ocurren en el mundo, que afectan de modo diferencial a la infancia, especialmente a los niños y niñas en los países en desarrollo. Cada infancia está regida por el hacer social en donde tiene lugar.

Esta profesión, tradicionalmente, ha sido asociada con connotaciones estereotipadas como femeninas, tales como: paciencia sin límites, vocación maternal, dedicación y ternura, amor y gusto por los niños y niñas, con un lenguaje de "infantilización" que permanece sin mucho cuestionamiento.

¿Qué se pide de este profesional en la actualidad? Las preguntas claves son: ¿Qué aprender para cambiar? ¿Qué cambiar para aprender? La realidad de la sociedad actual hace reflexionar que el educador debe buscar el perfeccionamiento permanente. Por otra parte, debe conducir a niños y niñas a abordar críticamente el conocimiento, a aceptar el cambio y la incertidumbre, porque esa es la realidad social actual. SSF 


\section{MASIFICACION DE UNIVERSIDADES EN LA FORMACION DEL EDUCADOR DE PÁRVULOS}

Las educadoras de párvulos en distintas etapas de la historia, han sido consideradas como profesionales importantes e irremplazables para la infancia de nuestro país.

Mirando la historia de la Educación Parvularia chilena, se recuerda a doña Amanda Labarca, Consejera de la Universidad de Chile y Presidenta de la Asociación de Mujeres Universitarias, quien presentó a esta Asociación, orientado por la pedagoga española Matilde Huici N., el plan de fundación de una Escuela Especial para formar educadoras dedicadas exclusivamente a niños entre los dos y seis años, que en Chile se venía llamando "la edad abandonada". Es así como con gran entusiasmo se acogió la idea y con el apoyo del Rector Don Juvenal Hernández Jaque, se abrió la Escuela de Educadoras de Párvulos en la Universidad de Chile en el año 1944, en Santiago de Chile.

Posteriormente (1968 - 1971), se abren las Carreras de Educadoras de Párvulos a lo largo del país: Arica, Iquique, Antofagasta, La Serena, Valparaíso, Talca, Chillán, Osorno. Se destaca también la creación de la carrera de formación de Educadores de Párvulos en la Universidad de Concepción en el año 1966, bajo la dirección de la Sra. Ivonne Fontaine P. Más adelante fue creada la Escuela en Educación Parvularia en la Pontificia Universidad Católica de Chile en el año 1972. Posteriormente, se crea la carrera en la Universidad Austral de Chile en Valdivia, en el año 1974.

El enfoque de la Educación Parvularia en Chile comienza a cambiar con las prácticas finales de las alumnas de la Universidad de Chile, re significando a la infancia desde el plano académico curricular, lo que desafiaba a las universidades a preparar a las alumnas de manera adecuada para que se desempeñaran en todos los escenarios donde se pudiera atender a la primera infancia; jardines infantiles, poblaciones, iglesias, hospitales, industrias, 
sanatorios, salitreras, entre otros, ampliando el ámbito de acción de la Educación Parvularia más allá de la escuela, como también extendiendo el rango de edad de atención desde el nacimiento hasta los seis años.

La modernización de la Ley de las Salas Cuna y la Ley de Guarderías y Jardines, concluyó en 1970 con la promulgación de la ley 17.301, de abril de 1970 que creó la Junta Nacional de Jardines Infantiles, lo que fue apoyado por todos los sectores políticos, movilización de estudiantes, madres trabajadoras y pobladoras.

Como consecuencia, se produjo una alta demanda de profesionales, lo que originó la necesidad de formación de Educadores de Párvulos a través de la Universidad de Chile y aquellas ya creadas para dar cumplimiento a la educación de calidad de la primera infancia en los diferentes sectores a lo largo del país.

Una segunda instancia histórica revela una masificación en la formación de educadores de párvulos. Surgió en los años 80 el sistema de educación que se modificó con las siguientes acciones: municipalización de la enseñanza en Chile, subvención por alumno, liberación del mercado laboral de los profesores, reestructuración de la educación superior y la Ley Orgánica Constitucional de Enseñanza de 1990.

En 1981 se experimentó una decisiva reestructuración legal, donde se distinguieron tres tipos de centros educativos; las universidades, los institutos profesionales y los centros de formación técnica. A las universidades se le asignaron 12 carreras de alto prestigio, mayor duración y reconocimiento que las carreras de educación. A los institutos, carreras de duración de formación de cuatro o cinco años, y a los centros de formación técnica, carreras de no más de dos años. Otra decisión fue que se dieron amplias facultades para la fundación de universidades, institutos y centros privados de educación.

Mirando el sistema actual de educación superior son, aproximadamente, 50 universidades las que forman educadoras de párvulos en el país, las cuales no 
aseguran una formación de calidad, dado que muchas de ellas, no cuentan con los recursos académicos para su funcionamiento a cabalidad.

Bajo esta realidad, la pregunta es ¿resulta una ventaja o desventaja esta masificación?

Siempre que existen instancias de discusión o conversación entre diferentes egresados de Educación Parvularia, resultará un beneficio poder intercambiar experiencias de estudios como una oportunidad de retroalimentación y enriquecimiento profesional de cada educador de párvulos, tomando en cuenta que cada titulado tenga la posibilidad, tiempo y disponibilidad de difundir su aprendizaje, sin embargo, esto no es una situación que se dé a menudo.

Por tanto, la variedad de mallas curriculares y enfoques que tiene cada institución educativa, revela la apropiación de diferentes perfiles de educadores, con competencias basadas en la práctica, en la teoría, en la reflexión u otras. Cada universidad tiene su propio sello en la formación de educadores de párvulos, el que no siempre responde, a cabalidad, las necesidades e intereses de niños y niñas en formación.

\section{CONDICIONES PARA LASI LOS EDUCADORES DE PÁRVULOS EN LA POLITICA NACIONAL DOCENTE EN CHILE}

Existen iniciativas legales que generan grandes expectativas en quienes podrían ser beneficiados con ellas. No es un problema menor que Chile se encuentre hoy abordando uno de los grandes desafíos para dar un mejor ordenamiento y validez a la labor docente en cada rincón de nuestro largo territorio, en cada establecimiento educativo donde se desempeñan profesores y profesoras $y$, especialmente, educadores y educadoras de párvulos, tanto en niveles correspondientes a primer nivel de transición (con niños y niñas de 4 a 5 años), 
segundo nivel de transición ( de 5 a 6 años), como en niveles de sala cuna (con niños de 0 a 2 años) y niveles medios menor ( 2 a 3 años) y mayor (3 a 4 años).

Hay acuerdo con la iniciativa de generar una carrera docente para regular un sistema que sea equitativo, ordenado, justo, que responda a las necesidades de quienes han asumido esta profesión, que motive a grandes talentos a seguir el camino de la educación y la formación humana, a quienes se les reconozca la importancia de su rol en la sociedad y en el futuro de nuevas generaciones. Debe, entonces, este mecanismo incentivar a la mejora permanente, la capacitación y perfeccionamiento en quienes educan.

La importancia de la Educación Parvularia es reconocida por todos: investigadores, estudiosos, profesionales de distintas especialidades, dado que es la base de la formación personal e implica la posibilidad del desarrollo de capacidades y competencias que se presentan en germen. En gran parte, el futuro de los niños y niñas depende de la Educación Parvularia, de la educación que reciba en sus primeros años de vida, en etapa de párvulo.

Por lo anteriormente expuesto, llama la atención que en las políticas públicas no quede claro como se considera a estas profesionales y no se les valora como se hace con los profesores y profesoras de los otros sistemas educativos. Vale decir, no se ve en forma clara el cómo y cuándo serán reconocidas sus capacidades y autonomía en el ejercicio de la profesión que ameriten asumir nuevas o más complejas funciones, como tampoco cuando serán objeto de reales mejoras en lo económico y en las condiciones laborales.

Por otra parte, en el plano de las mentorías, éstas deben ser ejecutadas por especialistas del mismo nivel educativo, dado que se trata de una función que requiere una mayor experiencia y especialización en un determinado nivel y ámbito educacional, debiendo estar estatuidas las condiciones para ejercer este 
tipo de funciones. Es de justicia reconocer la experiencia de las profesionales que han estado en determinada función para que se hagan cargo de nuevas demandas pedagógicas.

Tampoco está bien definido en la ley cómo las educadoras de párvulos de las instituciones que, tradicionalmente, han atendido la mayor cantidad de párvulos en Chile, llámese JUNJI, Integra y aquellos que funcionan con fondos aportados por JUNJI, se les incorporará a esta política nacional docente.

Es importante señalar el escaso número de alternativas de evaluación en cuanto a la docencia presentadas por las nuevas leyes. Se considera el portafolio, conocimientos disciplinares y experiencia, no obstante existen otras formas de evaluación del desempeño profesional que son más integradoras. Entre ellas, la supervisión educacional directa.

Es dable señalar lo acertado que ha sido establecer mayores condiciones de ingreso a los postulantes de las carreras de pedagogía, entre ellos el requisito de un puntaje mínimo de 500 puntos (PSU) a inicio de sus estudios profesionales y sujeto a mayores exigencias futuras

Del mismo modo cabe destacar el acierto de establecer la acreditación obligatoria de las carreras de formación de educadores y si ésta no se da en un plazo establecido, la supresión de esa determinada carrera.

La posible marginación o demora en incorporarse a la Política Nacional Docente de las educadoras de párvulos de organismos que atienden niños vulnerables, podría implicar un grave peligro, esto porque las profesionales recién tituladas u otras con mayor experiencia, optarían por incorporarse a aquellos establecimientos que tienen carrera docente, desestimando a aquellos que no la tienen, lo que significaría una baja en la calidad de atención educativa en los niños y niñas más necesitados. 


\section{BIBLIOGRAFÍA}

Estatutos Colegio Educadores Ed. Juan Vargas, Santiago, Chile.

de Párvulos de Chile AG, 1977.

Marchesi Alvaro, 2013.

Conferencia: "Educación de Calidad en la Primera Infancia y Desarrollo Profesional Docente". Edificio Gabriela Mistral. Santiago, Chile.

MINSAL 2008.

Chile Manual para el Apoyo y Seguimiento del

Desarrollo Psicosocial de los Niños y Niñas de 0 a 6 años.

Pramling Ingrid, 2005.

Formación de Personal para la Primera Infancia en el Siglo XXI ¿Estamos preparándonos adecuadamente? OMEP Chile, Santiago. 